Tuning the onset voltage of resonant tunneling through InAs quantum dots by growth parameters

I. Hapke-Wurst, U. Zeitler, U. F. Keyser, R. J. Haug, K. Pierz, and Z. Ma

Citation: Appl. Phys. Lett. 82, 1209 (2003); doi: 10.1063/1.1555712

View online: https://doi.org/10.1063/1.1555712

View Table of Contents: http://aip.scitation.org/toc/apl/82/8

Published by the American Institute of Physics

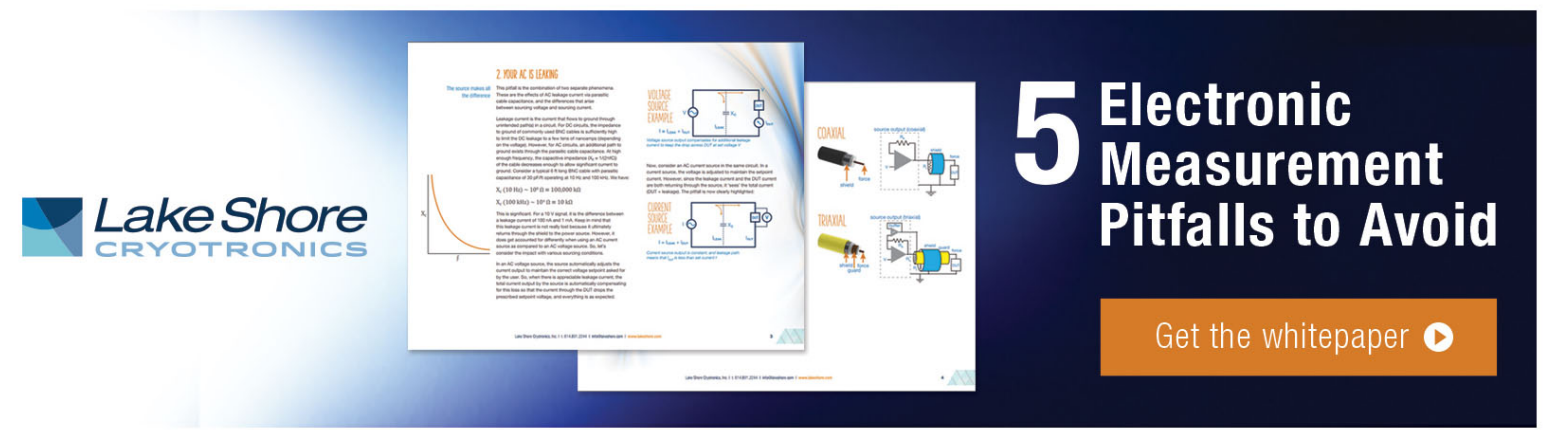




\title{
Tuning the onset voltage of resonant tunneling through InAs quantum dots by growth parameters
}

\author{
I. Hapke-Wurst, ${ }^{\text {a) }}$ U. Zeitler, ${ }^{\text {b) }}$ U. F. Keyser, and R. J. Haug \\ Institut für Festkörperphysik, Universität Hannover, Appelstr. 2, 30167 Hannover, Germany \\ K. Pierz and Z. Ma \\ Physikalisch-Technische Bundesanstalt Braunschweig, Bundesallee 100, 38116 Braunschweig, Germany
}

(Received 11 October 2002; accepted 3 January 2003)

\begin{abstract}
We investigated the size dependence of the ground-state energy in self-assembled InAs quantum dots embedded in resonant tunneling diodes. Individual current steps observed in the currentvoltage characteristics are attributed to resonant single-electron tunneling via the ground state of individual InAs quantum dots. The onset voltage of the first step observed is shown to decrease systematically from $200 \mathrm{mV}$ to 0 with increasing InAs coverage. We relate this to a coverage-dependent size of InAs dots grown on AlAs. The results are confirmed by atomic force micrographs and photoluminescence experiments on reference samples. () 2003 American Institute of Physics. [DOI: 10.1063/1.1555712]
\end{abstract}

Recently, self-assembled InAs quantum dots (QDs) grown in the Stranski-Krastanov mode have attracted much interest as a nanostructure with interesting electronic properties. In particular, their well defined optical characteristics as investigated in numerous photoluminescence experiments ${ }^{1}$ may be used for application in optical and optoelectronic devices such as QD laser and memories. ${ }^{2}$

Until the present only a few experiments directly access the electronic transport through InAs QDs by means of resonant tunneling through QDs embedded single-barrier tunneling diodes. ${ }^{3-8}$ Although typically $10^{5}-10^{6}$ QDs are present in the macroscopic devices used in such experiments, resonant single-electron tunneling through individual QDs was observed. This means that only a small proportion of the QDs in a tunneling structure contribute to the tunneling current, a much larger one is electrically inactive. As a consequence, a correlation between the experimentally observed single-QD features and the properties of the macroscopic QD ensemble is far from being straightforward in such type of experiments.

In this letter we will show that even in macroscopic diodes we can nevertheless relate the onset voltage for resonant single-electron tunneling through an individual QD to the ensemble properties of all QDs which can be thoroughly controlled by growth parameters. In particular, with increasing InAs coverage we find a systematically decreasing onset voltage for the first current step related to single-electron tunneling through the energetically lowest lying electronically accessible InAs QD. We relate this to an increasing quantum dot size with increasing coverage leading to a lower ground state energy. Our results are additionally supported by photoluminescence experiments and atomic force micrographs on reference samples. These findings may open up a way to control the properties of single-electron devices based on resonant tunneling via QDs.

\footnotetext{
${ }^{a}$ Present address: Infineon Technologies, Dresden, Germany.

${ }^{b}$ Present address: Research Institute for Materials, High Field Magnet Laboratory, Toernooiveld 1, 6525 ED Nijmegen, The Netherlands.
}

The resonant-tunneling diode structures were grown by molecular-beam epitaxy (MBE) on a 2 in. $n^{+}$-doped GaAs (100) substrate $\left.\left(n^{+}=2 \times 10^{24} \mathrm{~m}^{-3}\right)\right)^{7,8}$ A $n$-GaAsbackcontact of $1 \mu \mathrm{m}$ thickness with graded Si doping followed by an undoped GaAs prelayer of $15 \mathrm{~nm}$ and a $5 \mathrm{~nm}$ AlAs barrier was deposited at a substrate temperature $T_{S}=600^{\circ} \mathrm{C}$. During a growth interruption of $60 \mathrm{~s} T_{S}$ was lowered to $520^{\circ} \mathrm{C}$ and the substrate rotation was stopped. Subsequently, with a slow growth rate of $0.05 \mathrm{ML} / \mathrm{s}$, nominally 1.8 monolayers (ML) InAs, as measured in the middle of the wafer, were deposited. The formation of selfassembled QDs was observed by the change of the reflective high-electron energy diffraction pattern after deposition of 1.6 ML of InAs.

Due to the geometric position of the In effusion cell with respect to the nonrotating substrate an actual gradient ranging from 1.55 to $2.05 \mathrm{ML}$ of InAs coverage is present across the wafer. On the parts of the wafer where the InAs coverage $x$ exceeds 1.6 ML we observe the formation of selfassembled QDs in atomic force microscopy (AFM) images on comparable reference samples. On the remaining parts of the wafer $(x<1.6 \mathrm{ML})$ only a two-dimensional InAs wetting layer with no self-assembled QDs is formed.

After the InAs growth and a subsequent growth interruption during $10 \mathrm{~s}$ the wafer was rotated again and the QDs were covered with a 5-nm-thick AlAs barrier. Simultaneously $T_{S}$ was slowly ramped up to $600{ }^{\circ} \mathrm{C}$. Finally, another $15 \mathrm{~nm}$ of undoped GaAs and an upper contact of $1 \mu \mathrm{m}$ graded $n$-GaAs were grown.

Pieces with different InAs coverage were cut from the wafer and tunneling diodes with standard AuGeNi contacts were fabricated on a $40 \times 40 \mu \mathrm{m}^{2}$ area by wet-chemically etching. Each of these diodes contains approximately 5 $\times 10^{9}$ InAs QDs. Reference wafers for AFM and photoluminescence (PL) measurements were grown under similar growth conditions. For the AFM experiments a wafer with uncovered QDs grown on $20 \mathrm{~nm}$ of AlAs was used. An identical wafer, additionally capped by $20 \mathrm{~nm}$ AlAs and $10 \mathrm{~nm}$ GaAs, was grown for the use in PL experiments. 


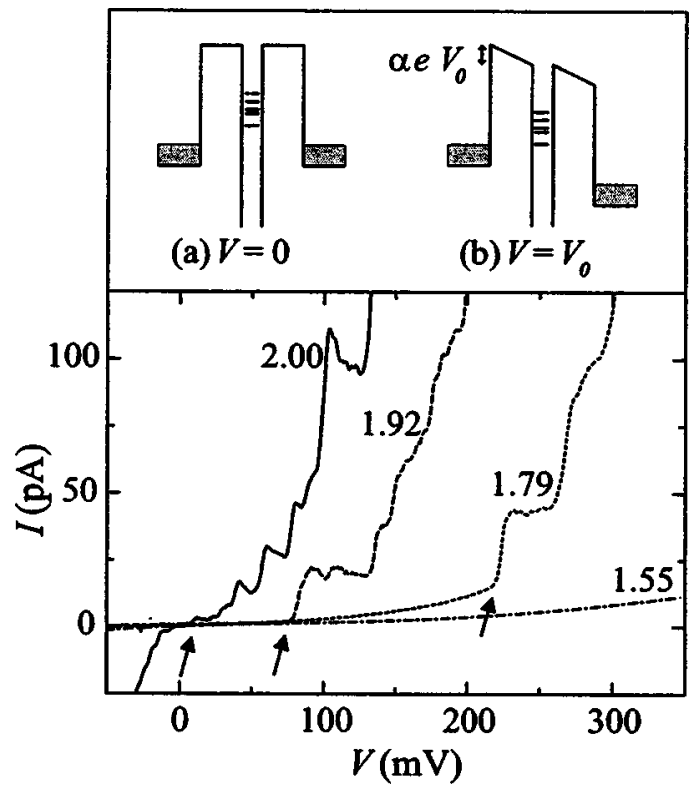

FIG. 1. Top: Schematic band structure of a tunneling diode at zero bias (a) and at finite bias where resonant tunneling through the electronically accessible InAs QD sets in (b). The levels between the electrodes denote the energetic positions of the ground states of individual, electronically accessible InAs quantum dots. Bottom: Current-voltage characteristics of samples with different InAs coverages. The numbers indicate the nominal number of monolayers of InAs deposited. The arrows mark the position of the current steps occurring whenever resonant tunneling through the energetically lowest lying InAs QD sets in [sketch (b) on the top panel].

The layer structure of the resonant-tunneling diodes is designed such that a three-dimensional electron gas with a Fermi energy $E_{F}$ of about $15 \mathrm{meV}$ above the conduction band is formed in the GaAs contact. ${ }^{8}$ As sketched in the top panel of Fig. 1 the QD ground states are generally situated above $E_{F}$ at zero bias. When increasing the bias voltage these states move towards lower energies with respect to $E_{F}$, and, whenever $E_{F}$ comes into resonance with such a QD level a step in the $I-V$ characteristics appears, see Fig. 1(b).

In the bottom panel of Fig. 1 we show such typical lowbias $I-V$ curves for diodes with different InAs coverages measured at $4.2 \mathrm{~K}$. In the structure with the lowest InAs coverage of 1.55 ML no QDs have formed and, consequently, a structureless, roughly exponential increase of the tunneling current is observed. In contrast, diodes with InAs coverages of more than 1.6 ML display step-like increasing $I-V$ characteristics which we assign to the resonant tunneling through individual InAs QDs. The first step in each $I-V$ curve appears at an onset voltage $V_{0}$, marked by the arrows in Fig. 1. With increasing InAs coverage $V_{0}$ systematically moves down towards zero. This shows that the energy levels of the QDs accessed by tunneling continuously shift to lower energies when the InAs coverage is increased. ${ }^{9}$

The onset voltage $V_{0}$ is in fact directly related to the ground state energy $E_{0}$ of the first dot accessible to resonant tunneling, $\alpha e V_{0}=E_{0}$, where $E_{0}$ is measured with respect to the Fermi energy in the emitter at zero bias. The lever factor $\alpha$ accounts for the fact that only a part of the total voltage applied drops between the emitter and the QD. Since we observe an abrupt onset of current steps at a finite voltage it is reasonable to assume that the first tunneling steps can be related to dots with a relatively low ground state energy situ-

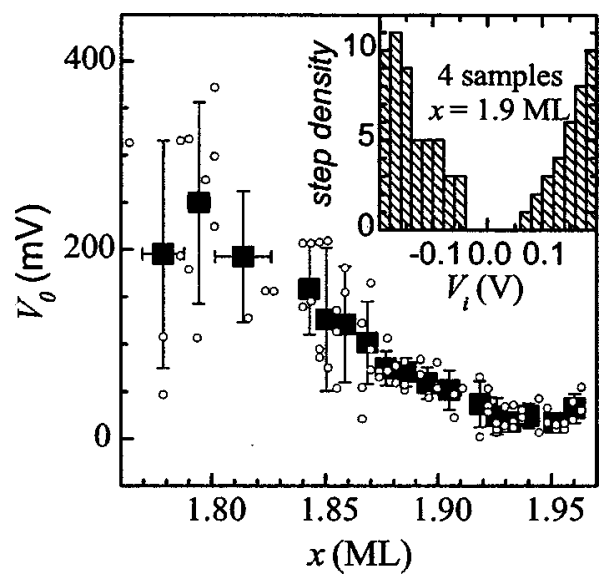

FIG. 2. Onset voltage $V_{0}$ of the InAs QD accessible to tunneling as a function of the InAs coverage. The small open dots correspond to individual samples. The solid rectangles represent an averaging over five samples with similar InAs coverage, the error bars show the statistical error of the average. The inset shows a histogram of the step density, defined as the number of steps observed in a $20 \mathrm{mV}$ interval, summed up over four samples with $x=1.9 \mathrm{ML}$, as a function of the corresponding onset voltages.

ated in the low-energy tail of the QD energy distribution. Subsequent steps then address dots with an energetically higher lying ground state.

The total number of dots per diode that participate in resonant tunneling can be roughly estimated by referring the total current at higher voltages to the current contribution of one single dot. Supposing a current contribution of roughly 20 pA (see, e.g., Fig. 1) for transport through one single dot a current of typically $1 \mathrm{nA}$ observed around $400 \mathrm{mV}$ yields a total number of about 50 dots electronically active at this bias voltage. Each dot contributes to the current in a bias range of $40 \mathrm{mV}{ }^{10}$ Using the total energetic width of the dot distribution as deduced from photoluminescence measurements (see later) we can extrapolate the 50 dots observed in a $40 \mathrm{mV}$ range to an upper bound of totally less than 1000 dots participating in resonant tunneling over the entire bias voltage range.

The systematic decrease of $V_{0}$ with increasing InAs coverage was verified by measuring totally 85 samples with InAs QDs. All the measured onset voltages are compiled in Fig. 2. In particular for InAs coverages $x>1.8 \mathrm{ML}$ a strong correlation between the onset voltage of the first current step and the InAs coverage is found. Such a correlation clearly shows that $V_{0}$ is strongly related to the macroscopic ensemble properties of the InAs quantum dots, and, in particular, to ground state energies $E_{0}$ decreasing with increasing coverage.

We have verified this statement with PL experiments on reference samples performed at $T=6 \mathrm{~K}$ under $\mathrm{cw}$ excitation with an $\mathrm{Ar}^{+}$laser $(514 \mathrm{~nm})$. As shown in Fig. 3(a) the position of the PL peak corresponding to the average energy of the ground-state transitions inside the InAs QDs indeed moves systematically towards lower energies when increasing the InAs coverage.

We have additionally investigated the energy distribution of the QDs accessed by resonant tunneling by systematically measuring four samples with a similar InAs coverage $x \approx 1.9$ ML over a broad voltage range. As can been seen in the inset of Fig. 2 the step density, defined as the number of steps in a 


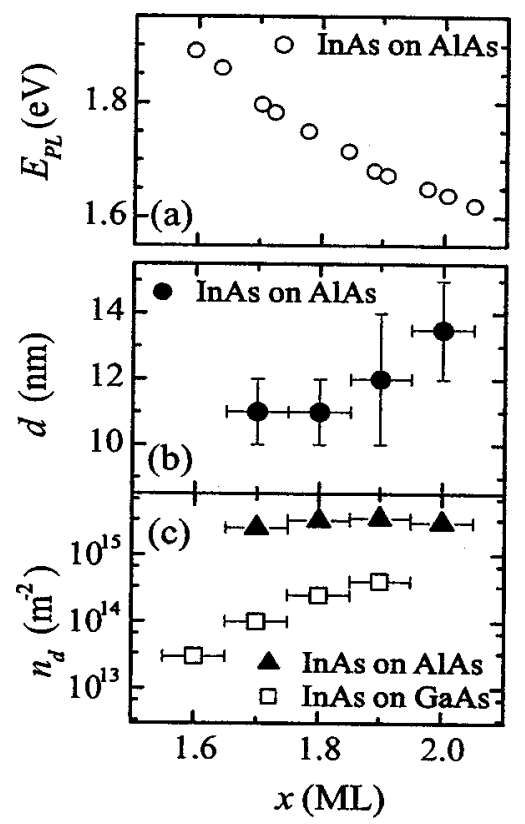

FIG. 3. (a) Energy of the PL peak assigned to the optical transition inside the InAs QDs as a function of the InAs coverage. (b) Average dot size of InAs QDs grown on AlAs. (c) InAs-QD density as a function of the InAs coverage for dots grown on AlAs (closed triangles) and on GaAs (open squares). The data are deduced from AFM experiments on reference samples.

$20 \mathrm{mV}$ interval summed up over all four samples, increases with an increasing absolute value of the bias voltage. This clearly proves that the first steps indeed do access the low energy tail of the QD distribution and subsequent steps access energetically higher lying states with energies moving towards the maximum in the energy distribution of the QDs.

The key point to understand Fig. 2 observed systematic tendency in the coverage dependence of the onset voltage for the first current step lies in the fact that the size of the dots grown on relatively rough AlAs is increasing when the InAs coverage is risen. ${ }^{11}$ In contrast, the size of InAs QDs grown on a much smoother GaAs surface does merely depend on the amount of InAs deposited. Such a size variation of the InAs QDs as a function of the InAs coverage can be verified independently by AFM, see Fig. 3(b). Indeed, the lateral QD dimensions increase from 10 to $14 \mathrm{~nm}$ in diameter when varying the InAs coverage from 1.6 to $2.0 \mathrm{ML}$. On the other hand, as shown in Fig. 3(c), the QD density of InAs dots grown on AlAs, $n_{\mathrm{QD}} \approx 3 \times 10^{15} \mathrm{~m}^{-2}$, is nearly independent on the InAs coverage $x$, whereas it strongly depends on $x$ for InAs QDs grown on GaAs. This observation shows that the growth of InAs QDs on AlAs (at the temperatures used in our experiments) is kinetically limited by a slower In adatom diffusion compared to the GaAs surface, which results into a fast nucleation of QDs at positions predefined by the surface roughness.

In conclusion, we have shown that the onset of resonant single-electron tunneling through self-assembled InAs QDs embedded in macroscopic tunneling diodes can be tuned between 0 and $0.3 \mathrm{~V}$ by adjusting the amount of InAs deposited during MBE growth of the corresponding device. This observation was explained by a systematic increase of the InAs QDs with increasing InAs coverage which can be related to the specific growth mechanism of QDs on AlAs. The energetic distribution of the dots involved in resonant tunneling was shown to correspond to that of the macroscopic ensemble. However, only a small relative proportion of InAs QDs were shown to participate in resonant tunneling, the rest are electronically inactive.

${ }^{1}$ See e.g., D. Bimberg, M. Grundmann, and N. N. Ledentsov, Quantum Dot Heterostructures (Wiley, Chichester, 1999).

${ }^{2}$ E. Biolatti, I. D’Amico, P. Zanardi, and F. Rossi, Phys. Rev. B 65, 075306 (2002), and references therein.

${ }^{3}$ I. E. Itskevich, T. Ihn, A. Thornton, M. Henini, T. J. Foster, P. Moriarty, A. Nogaret, P. H. Beton, L. Eaves, and P. C. Main, Phys. Rev. B 54, 16401 (1996).

${ }^{4}$ T. Suzuki, K. Nomoto, K. Taira, and I. Hase, Jpn. J. Appl. Phys., Part 1 36, 1917 (1997).

${ }^{5}$ M. Narihiro, G. Yusa, Y. Nakamura, T. Noda, and H. Sakaki, Appl. Phys. Lett. 70, 105 (1997).

${ }^{6}$ A. S. G. Thornton, T. Ihn, P. C. Main, L. Eaves, and M. Henini, Appl. Phys. Lett. 73, 354 (1998).

${ }^{7}$ I. Hapke-Wurst, U. Zeitler, H. W. Schumacher, R. J. Haug, K. Pierz, and F. J. Ahlers, Semicond. Sci. Technol. 14, L41 (1999).

${ }^{8}$ I. Hapke-Wurst, U. Zeitler, H. Frahm, A. G. M. Jansen, R. J. Haug, and K. Pierz, Phys. Rev. B 62, 12621 (2000).

${ }^{9}$ I. Hapke-Wurst, Ph.D. thesis, Hannover, 2002.

${ }^{10}$ A. Nauen, I. Hapke-Wurst, F. Hohls, U. Zeitler, R. J. Haug, and K. Pierz, Phys. Rev. B 66, 161303(R) (2002).

${ }^{11}$ P. Ballet, J. B. Smathers, and G. J. Salamo, Appl. Phys. Lett. 75, 337 (1999). 